\title{
Mucosa-reparing and microbiota-balancing therapeutic effect of Bacillus subtilis alleviates dextrate sulfate sodium-induced ulcerative colitis in mice
}

\author{
HUI-LU ZHANG ${ }^{1 *}$, WEN-SHUAI LI ${ }^{1 *}$, DIAN-NAN XU ${ }^{2 *}$, WAN-WEI ZHENG ${ }^{1}$, YI LIU ${ }^{1}$, \\ JIAN CHEN $^{1}$, ZHI-BING QIU ${ }^{1}$, ROBERT G. DORFMAN ${ }^{3}$, JUN ZHANG ${ }^{1}$ and JIE LIU ${ }^{1,2}$
}

${ }^{1}$ Department of Digestive Diseases, Huashan Hospital, Fudan University, Shanghai 200040; ${ }^{2}$ Department of Immunology, Institute of Biomedical Sciences, Fudan University, Shanghai 200032, P.R. China; ${ }^{3}$ MD Candidate, Class of 2018, Feinberg School of Medicine, Northwestern University, Chicago, IL 60611, USA

Received May 5, 2016; Accepted June 6, 2016

DOI: $10.3892 /$ etm.2016.3686

\begin{abstract}
Gut microbiota composition of patients with ulcerative colitis (UC) is markedly altered compared with healthy individuals. There is mounting evidence that probiotic therapy alleviates disease severity in animal models and patients with inflammatory bowel disease (IBD). Bacillus subtilisis, as a probiotic, has also demonstrated a protective effect in IBD. However, the therapeutic mechanism of its action has yet to be elucidated. In the present study, a dextrose sulfate sodium (DSS)-induced UC mouse model was used to investigate the role of B. subtilis in the restoration of gut flora and determine its effective dose. Mucosal damage was assessed by performing alcian blue staining, cytokine levels were analyzed by ELISA and microbiota composition was investigated using 454 pyrosequencing to target hypervariable regions V3-V4 of the bacterial 16S ribosomal RNA gene. The results demonstrated that a higher dose $B$. subtilisis administration ameliorated DSS-induced dysbiosis and gut inflammation by balancing beneficial and harmful bacteria and associated anti- and pro-inflammatory agents, thereby aiding intestinal mucosa recovery from DSS-induced injuries. These findings indicate that choosing the correct dose of $B$. subtilis is important for effective UC therapy. The present study also helped to elucidate the mechanisms of $B$. subtilis action and provided preclinical data for B. subtilis use in UC therapy.
\end{abstract}

Correspondence to: Professor Jie Liu, Department of Digestive Diseases, Huashan Hospital, Fudan University, 12 Middle Wulumuqi Road, Shanghai 200040, P.R. China E-mail: jieliu@fudan.edu.cn

*Contributed equally

Key words: Bacillus subtilis, ulcerative colitis, mouse

\section{Introduction}

Ulcerative colitis (UC), which is a subtype of inflammatory bowel disease (IBD) (1), is a chronic and debilitating condition that results in serious intestinal injuries. UC typically occurs as a result of inflammatory dysfunction (2). Patients with UC often exhibit intestinal barrier dysfunction, as well as microbiota and bacterial dysbiosis (3).

Although UC is common, its etiology remains poorly understood (4). In a previous study, epithelial barrier impairment was demonstrated to be associated with low-grade inflammation and dysbiosis as potential causative factors, and are associated with the severity of UC (2). Furthermore, in patients with UC, an increase in gut permeability has previously been associated with the altered expression levels or distribution of tight junction proteins, including occludin and zonula occludens-1 (ZO-1) (5). Therefore, increased intestinal permeability and the occurrence of dysbiosis may be the cause of UC-symptoms (6). The evidence that gut microbiota may have a role in the pathophysiology of UC provides a rationale for probiotic use, which has exhibited beneficial effects (7). However, the therapeutic mechanism of action for the effect of probiotics in UC has yet to be elucidated.

Probiotics are defined as live organisms that exert a health benefit on the host through diverse mechanisms. Bacillus subtilis is a type of probiotic tolerated by humans and animals (8). B. subtilis is hypothesized to affect the composition or function of the commensal, bacterial and host epithelia. Furthermore, it also influences immunological responses and restricts bacterial and lipopolysaccharide (LPS) translocation, and decreases visceral sensitivity (8). In recent clinical trials, probiotics have been widely used to treat disorders of the intestine (7). As enhancement of the intestinal barrier has been associated with the repair of mucosal injuries, the role of $B$. subtilis treatment in maintaining gut barrier integrity was investigated in the present study, due to its potential usage in the alleviation of UC mucosal injuries.

Changes in the gut microbiota have been associated with IBD, including alterations in the relative abundance of bacteria that are both beneficial and detrimental to gut health, and a 
decrease in the diversity of the microbiota $(9,10)$. Although previous reports have demonstrated the protective effect of B. subtilis in gut protection $(11,12)$, the impact of $B$. subtilis administration on gut microbiota alteration remains unknown. The present study aimed to elucidate the role of $B$. subtilis in the restoration of mucosa, determine its effective dose, and provide preclinical data for B. subtilis usage in UC therapy.

\section{Materials and methods}

Modeling of colorectal colitis in mice and treatment. Male C57 mice (body weight, $23 \pm 1 \mathrm{~g}$; 6 weeks old) were obtained from the Animal Center, Nanjing Drum Tower Hospital (Nanjing, China) and the in vivo experiment was performed in the same facility. Mice were maintained under controlled conditions $\left(25^{\circ} \mathrm{C}, 55 \%\right.$ humidity, $12 \mathrm{~h}$ light/dark cycle) and fed standard laboratory food. Mice were administered 3\% (wt/vol) dextrose sulfate sodium (DSS) (molecular weight, 35,000-44,000; MP Biomedicals, Inc., Aurora, OH, USA) via drinking water for seven days. Additionally, mice were treated daily with different reagents via gavage (catheter diameter, $1.2 \mathrm{~mm}$ ), including normal saline (NS; $\mathrm{n}=8$ ) or B. subtilis (R179; Beijing Hanmi Pharm Co., Ltd., Beijing, China) at a high $\left(1 \times 10^{9} \mathrm{CFU} /\right.$ mouse/day; $\left.\mathrm{n}=8\right)$ or low $\left(1 \times 10^{8} \mathrm{CFU} / \mathrm{mouse} /\right.$ day; $\left.\mathrm{n}=8\right)$ dosage until the end of the study. On day eight, mice were weighed and then sacrificed via ether exposure (200 mg/l; Shanghai National Medicine Group, Shanghai, China) in an airtight container in a biosafety cabinet. Colons were harvested, measured and fixed in $4 \%$ formalin for subsequent histological examination. Animal experiments were approved by the Ethics Committee of Medical Research, Huashan Hospital of Fudan University (Shanghai, China).

Assessment of colitis. Following the initiation of DSS treatment, daily changes in body weight and clinical signs of colitis, such as rectal bleeding, diarrhea and piloerection, were examined. The disease activity index consisted of scoring for rectal bleeding (0-4), as previously reported (13). Hemoccult SENSA (Beckman Coulter, Inc., Brea, CA, USA) was used to examine rectal bleeding.

Periodic acid-Schifflalcian blue staining. Alcian blue staining was performed according to a previous report (14). Tissue sections (6 $\mu \mathrm{m}$ thick) were immersed in $100 \%$ ethanol for $10 \mathrm{~min}$, rinsed in water for $10 \mathrm{~min}$, immersed in $3 \%$ acetic acid for 2 min and subsequently stained in $1 \%$ alcian blue $8 \mathrm{GX}$ in $3 \%$ acetic acid ( $\mathrm{pH} 2.5$ ) for $2.5 \mathrm{~h}$. To remove non-specific staining, $3 \%$ acetic acid and water was used to rinse the sections for $10 \mathrm{~min}$. Slides were subsequently oxidized in $1 \%$ periodic acid in water at room temperature for $10 \mathrm{~min}$, washed in water for $5 \mathrm{~min}$, immersed in Schiff's reagent for $10 \mathrm{~min}$, rinsed in water for $5 \mathrm{~min}$ and three times in $0.5 \%$ sodium metabisulphite prior to a final wash in water. To reveal O-acetylated oligosaccharides, sections were treated with $0.1 \mathrm{M} \mathrm{KOH}$ for $30 \mathrm{~min}$ and $1 \mathrm{mM}$ periodic acid prior to the Schiff reagent.

Immunofluorescence. Frozen tissue sections ( $6 \mu \mathrm{m}$ thick) were immunostained with 1:100 primary antibodies against ZO-1 (clonality, H-300; cat. no. sc-10804) and claudin (clonality, D-4; cat. no. sc-137121; species: mouse, rat, human, equine, canine, bovine, porcine) (both Santa Cruz Biotechnology, Inc., Santa
Cruz, CA, USA). Images were analyzed using a BIOREVO immunofluorescence microscope (Keyence Corp., Osaka, Japan). Each result was obtained from at least three separate experiments. Six mice per group were prepared for each experiment.

Measurement of intestinal permeability. Intestinal permeability was determined according to a previously described method (15). DSS-treated mice with high/low-dose B. subtilis or control saline ( $\mathrm{n}=5 ; 4$ days) were fasted for $12 \mathrm{~h}$ prior to oral gavage of disaccharide permeability probes $[100 \mathrm{mg} / \mathrm{ml}$ lactulose and $50 \mathrm{mg} / \mathrm{ml}$ mannitol (both Sigma-Aldrich; Merck Millipore, Darmstradt, Germany) dissolved in $2 \mathrm{ml}$ water] and urine was collected $12 \mathrm{~h}$ later. Urine volume was measured and the concentrations of lactulose and mannitol were determined by high-performance liquid chromatography with an NH2 column (Bischoff Chromatohraphy, Leonberg, Germany) and acetonitrile (70\%) based elution. The ratio of the amount of probe in urine to the amount administered as lactulose or mannitol recovery rate was calculated accurately. Intestinal permeability was evaluated as a ratio of lactulose recovery rate to mannitol recovery rate.

Measurement of serum cytokines and endotoxin. On the 4th day following DSS treatment, mice were anesthetized with ether (200 mg/l; Shanghai National Medicine Group) in an airtight container within a biosafety cabinet and blood was collected from the retrobulbar venous plexus using pyrogen-free heparinized syringes. Cytokine [interleukin (IL)-10, IL-12 p70, IL-17A, and IL-23] levels were analyzed by ELISA according to the manufacturer's protocol (R\&D Systems, Inc., Minneapolis, MN, USA). Plasma endotoxin was measured using a Limulus amebocyte lysate pyrogen test kit (Xiamen Houshiji, Ltd., Xiamen, China; cat. no. KC48).

Short-chain fatty acid (SCFA) assay. Fresh mice fecal samples were collected from the cages, weighed and stored at $-80^{\circ} \mathrm{C}$. Fecal samples were mixed with distilled water and centrifuged $(2,500 \mathrm{x} \mathrm{g})$. The supernatant was removed, filtered and mixed with ether and sulfuric acid. Following centrifugation $(2,500 \mathrm{x} \mathrm{g})$, the ether layer was collected and measured in an Agilent 6890N gas chromatograph machine (Agilent Technologies, Inc., Santa Clara, CA, USA) to determine the total SCFA concentrations.

Microbiological analysis of mice fecal samples. Microbiota composition was assessed by 454 pyrosequencing (GS FLX TI technology, Genoscreen, Lille, France) targeting the V3-V4 region of the bacterial 16S rRNA gene (V3, forward 5'-TAC GGRAGGCAGCAG-3' and V4 reverse 5'-GGACTACCA GGGTATCTAAT-3'). Sequences were binned for a minimal sequence length of $300 \mathrm{pb}$, a minimal base quality threshold of 30 cycles and a maximum homopolymer length of 6 cycles. Resulting sequences were assigned to different taxonomic levels, from phylum to genus using the Ribosomal Database Project (16). Sequences were further clustered into operational taxonomic units (OTUs) or phylotypes at $97 \%$ of identity using the Quantitative Insights into Microbial Ecology pipeline and CD-HIT $(17,18)$. OTUs were assigned to their closest taxonomic neighbors and relative bacterial species using Seqmatch 
A

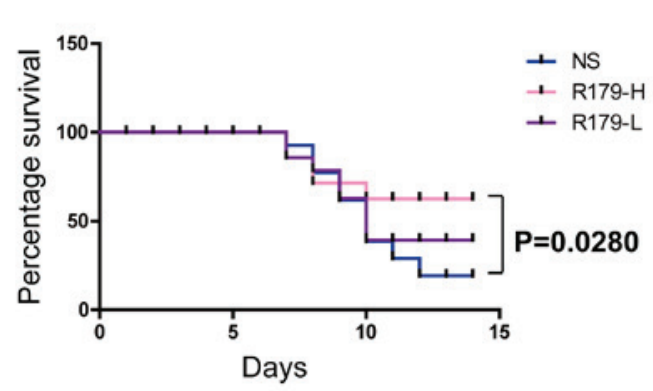

C

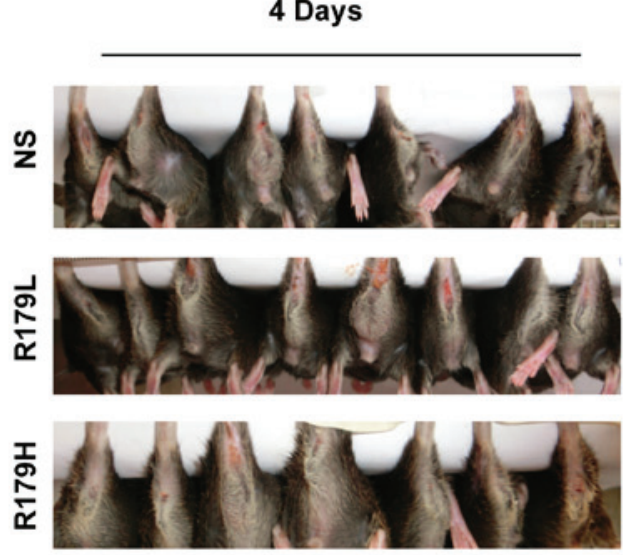

E

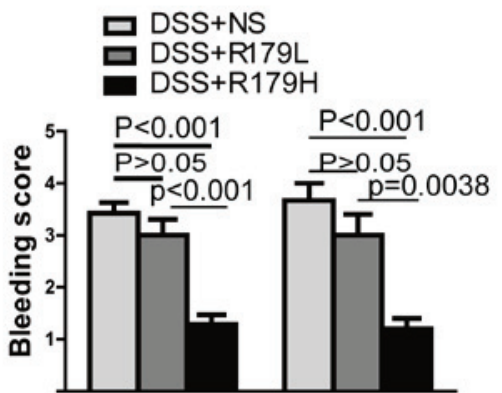

B

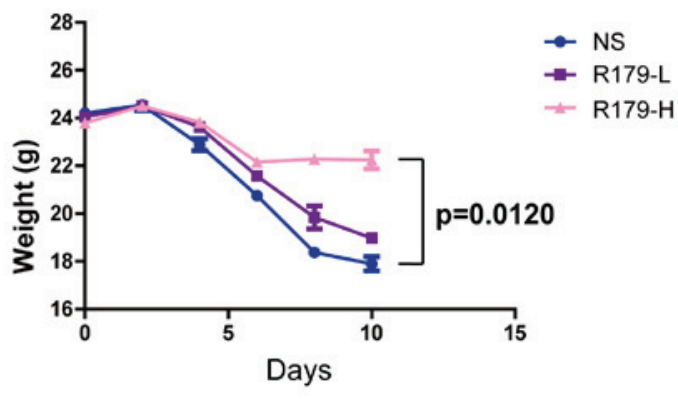

D

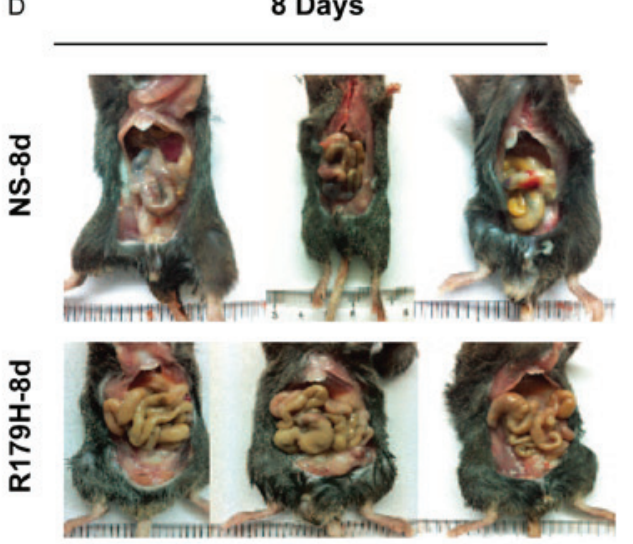

F

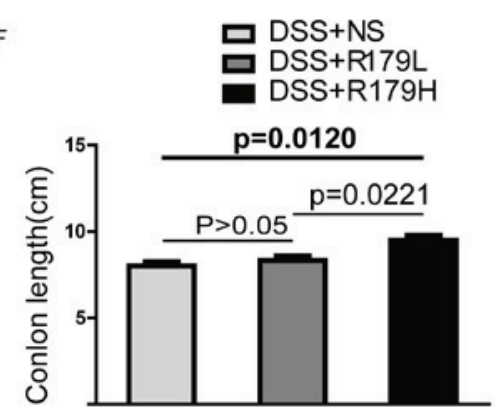

Figure 1. Bacillus subtilis alleviates DSS-induced lethality and intestinal injuries in mice. (A) Percentage survival rate over time; (B) change in body weight over time; representative image of (C) degree of gut damage and (D) colon length after mice were treated for 8 days; (E) bleeding scores after mice were treated for 4 days; and (F) colon length after mice were treated for 8 days. DSS, dextrose sulfate sodium; NS, normal saline; R179-L, low-dose B. subtilis; R179-H, high-dose B. subtillis.

(Michigan State University, East Lansing, MI, USA) and Blastall (National Centre for Biotechnology Information, Bethesda, MD, USA).

Statistical analysis. Data were expressed as the mean \pm standard error of the mean. Differences were analyzed using Student's $t$-test, Chi-square test, or one-way analysis of variance with Tukey's post-hic test for multiple group comparison. $\mathrm{P}<0.05$ was considered to indicate a statistically significant difference.

\section{Results}

B. subtilis alleviates DSS-induced lethality and intestinal injuries in mice. To examine the role of $B$. subtilis in the amelioration of $\mathrm{UC}$ in vivo, mice were initially exposed to a lethal dose of $4 \%$ DSS (20 ml/d), a pharmacological agent used to induce UC that also causes severe secondary symptoms. Mice were subsequently treated orally with either a high- or low-dose of B. subtilis preparation, or phosphate-buffered saline for control at eight days post-administration. The results indicated that the high dose of $B$. subtilis solution protected mice from the lethal effect of DSS-induced UC (Fig. 1A). At the end of the study, $\sim 50 \%$ survival of probiotic-treated mice was observed. In contrast, only $\sim 1 / 3$ of mice who received normal saline survived for the same period as the high-dose probiotic-treated group and this difference was significant ( $\mathrm{P}=0.0280$; Fig. 1A). In addition, a high-dose of $B$. subtilis administration significantly ameliorated weight loss compared 
A
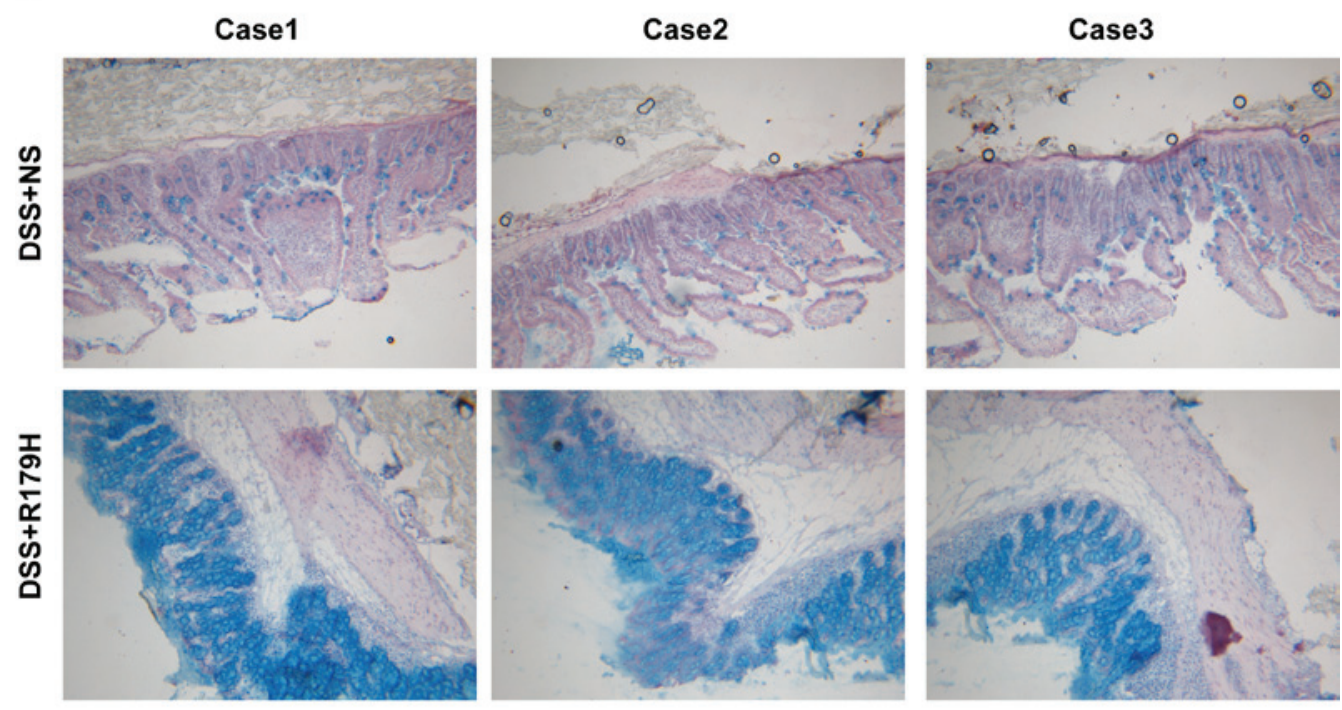

B

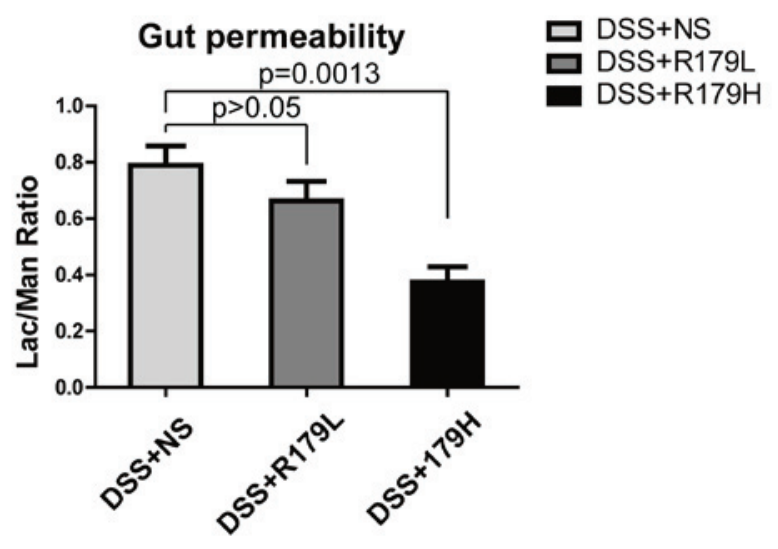

Figure 2. Bacillus subtilis protects mouse intestinal mucosal from DSS-induced damage. (A) Mucin was detected by alcian blue staining after mice were treated for 4 days. (B) Detection of intestinal permeability after mice were treated for 4 days. DSS, dextrose sulfate sodium; NS, normal saline; R179L, low-dose B. subtilis; R179H, high-dose B. subtillis.

with the normal saline group ( $\mathrm{P}=0.0120$; Fig. 1B). As DSS can promote intestinal damage, such as hematochezia and intestinal bleeding, the anuses and intestinal tracts of the three groups were examined. For mice in the high-dose B. subtilis-treated group, the anus and intestinal tract exhibited reduced bleeding and anabrosis than the control and low dose groups (Fig. 1C and D). Mice in the control and low-dose groups suffered more severe colon necrosis and shorter colons compared with the high-dose B. subtilis-treated group (Fig. 1E and F). These results indicate that high-dose $B$. subtilis administration alleviates DDS-induced colon damage and that $B$. subtilis induces a dose-dependent effect.

B. subtilis protects against DSS-induced intestinal mucosal damage in mice. DSS induces UC by causing serious intestinal mucosal damage (19). In accordance with the findings mentioned, low or high doses of B. subtilis may attenuate the symptoms of DSS-induced UC. In light of this, it was hypothesized that $B$. subtilis may produce its effect by protecting the intestinal mucosa from damage and by reinforcing its repair. To test this hypothesis, alcian blue staining was conducted to determine how the intestinal mucosa reacted to the administration of $B$. subtilis following DSS treatment. As shown in Fig. 2A, mice treated with high-dose B. subtilis exhibited increased expression levels of mucins compared with the control group, which indicated repair of the colon mucosa. These results suggest that the high dose of the $B$. subtilis probiotic promoted the restoration of intestinal mucosa.

To further test this hypothesis, intestinal permeability was measured, as DSS-induced intestinal mucosa damage may lead to an increase in intestinal permeability. The results of the present study demonstrated that intestinal permeability was recovered in the B. subtilis-treated group (Fig. 2B). This further supports the hypothesis that treatment with $B$. subtilis may protect intestinal mucosa from DSS-induced damage and attenuate the inflammatory reaction.

B. subtilis helps to restore tight junctions. The tight junction complex of the intestinal mucosa is considered to be the first 'firewall' for gut immunity (20). ZO-1 and claudins are two large families of the tight junction complex. To further explore whether B. subtilis was able to repair DSS-induced damage to the tight junctions, the intestines of mice treated with or without $B$. subtilis were harvested and probed with 
A

Claudine IF staining

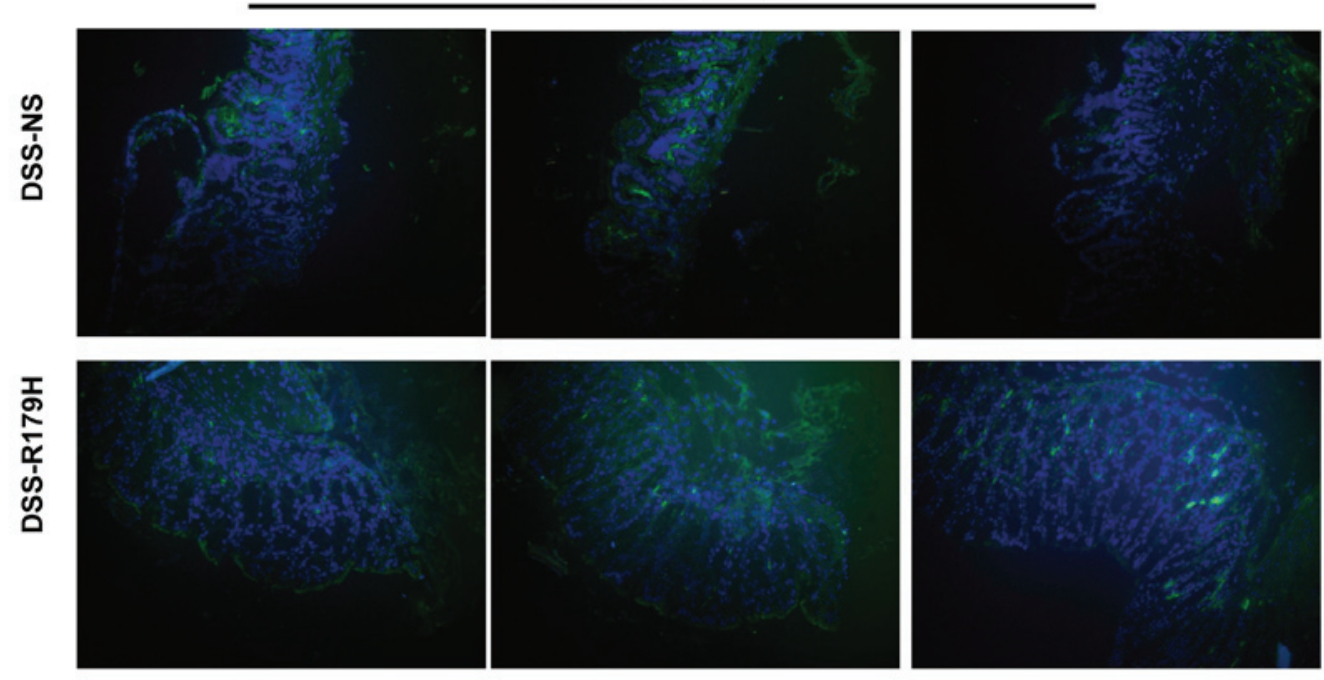

B

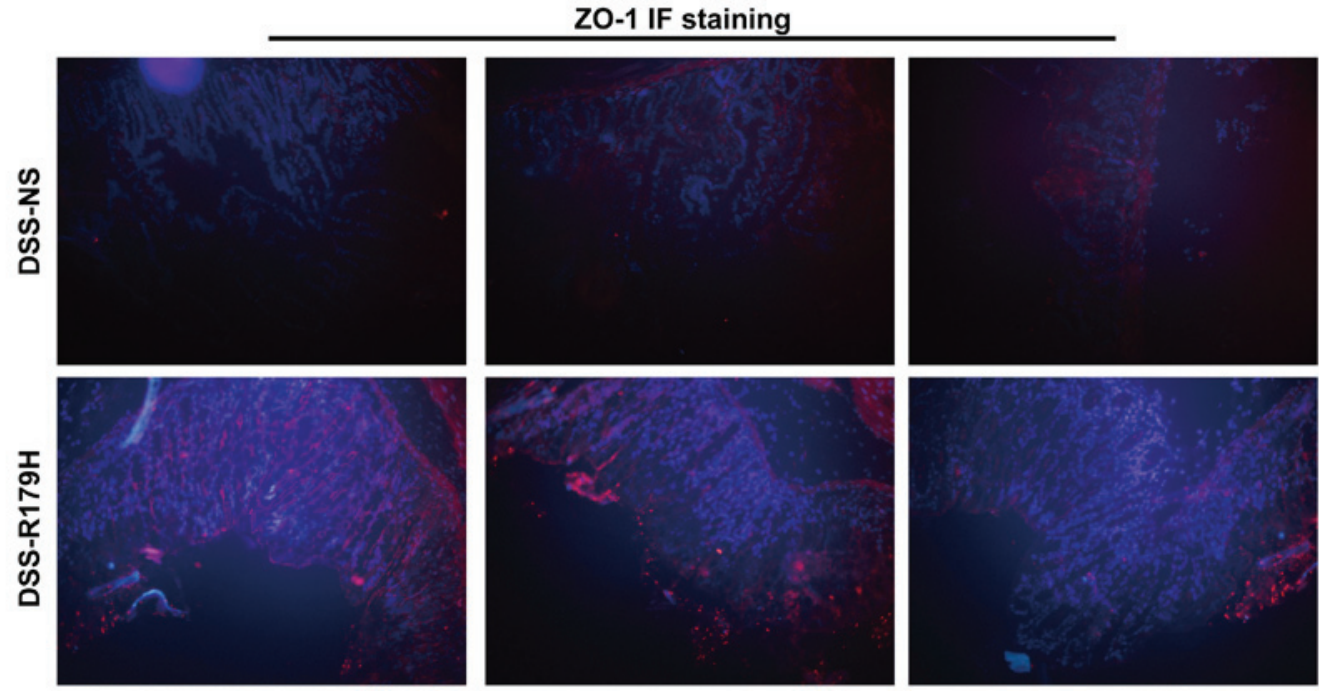

Figure 3. Bacillus subtilis helps restore tight junctions damaged by DSS. IF staining with the primary antibody (A) claudin and (B) ZO-1. IF, immunofluorescence; DSS, dextrose sulfate sodium; NS, normal saline; R179H, high-dose B. subtillis; ZO-1, zona occludens-1.

ZO-1 and claudins. The samples were then observed under a confocal laser scanning microscope, and the results demonstrated that the two tight junction-associated markers increased following high-dose B. subtilis treatment (Fig. 3A and $\mathrm{B})$. This indicated that $B$. subtilis was involved in the repair process of DSS-induced mucosal damage and restored the mucosal tight junction complex.

B. subtilis administration alleviates systemic inflammation upon DSS treatment. Restoration of intestinal permeability may be related to the relief of the inflammation reaction (21). Therefore, the effect of $B$. subtilis administration on gut inflammation was explored using ELISA. An increase in plasma cytokines has previously been reported, including IL-12, IL-17 and IL-23, whereas IL-10 decreased in IBD (22). In the present study, the mean plasma levels of IL-12, IL-17 and IL-23 in the high-dose $B$. subtilis group were significantly reduced $(\mathrm{P}=0.0411,0.0087$ and 0.0152 , respectively) and the mean IL-10 plasma levels were significantly increased
$(\mathrm{P}=0.0450)$ (Fig. 4) compared with the NS group. However, low-dose B. subtilis treatment produced a less-marked effect.

B. subtilis administration balances anti-and pro-inflammatory factors in the gut of mice. Gut microbiota is a primary source of LPS endotoxin, which is a damage-associated pathogen that promotes gut inflammatory reactions and systemic inflammation $(23,24)$. Plasma LPS of mice was tested and a difference in LPS was detected between the high-and low-dose B. subtilis-treated groups and the control group. These results indicated a significant decrease of LPS concentration in the probiotic group with the administration of high-dose B. subtilis compared with the control group $(\mathrm{P}<0.001$; Fig. 5A).

SCFAs have anti-inflammatory functions via interaction with $\mathrm{G}$ protein-coupled receptor 43 and have been demonstrated to induce pro-inflammatory cytokines in various models of colitis $(25,26)$. The concentration of total SCFAs was significantly higher in the high-dose B. subtilis group 
A

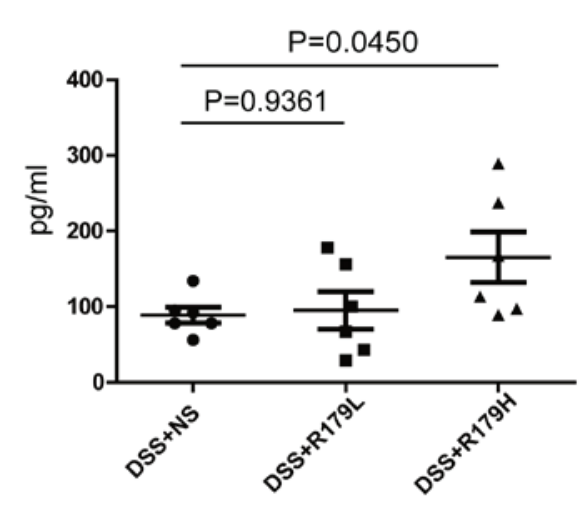

C

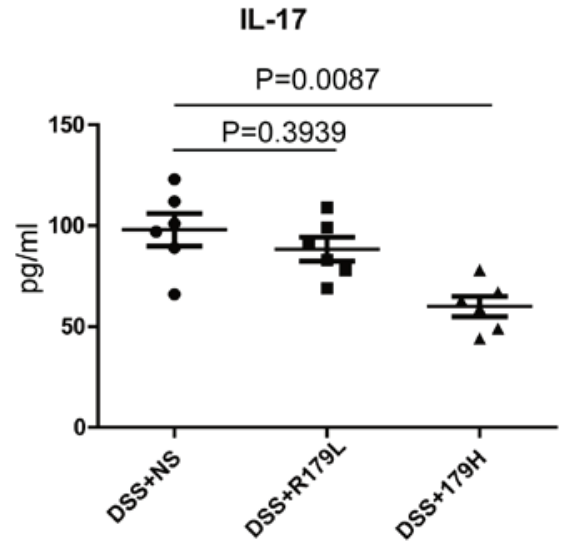

B
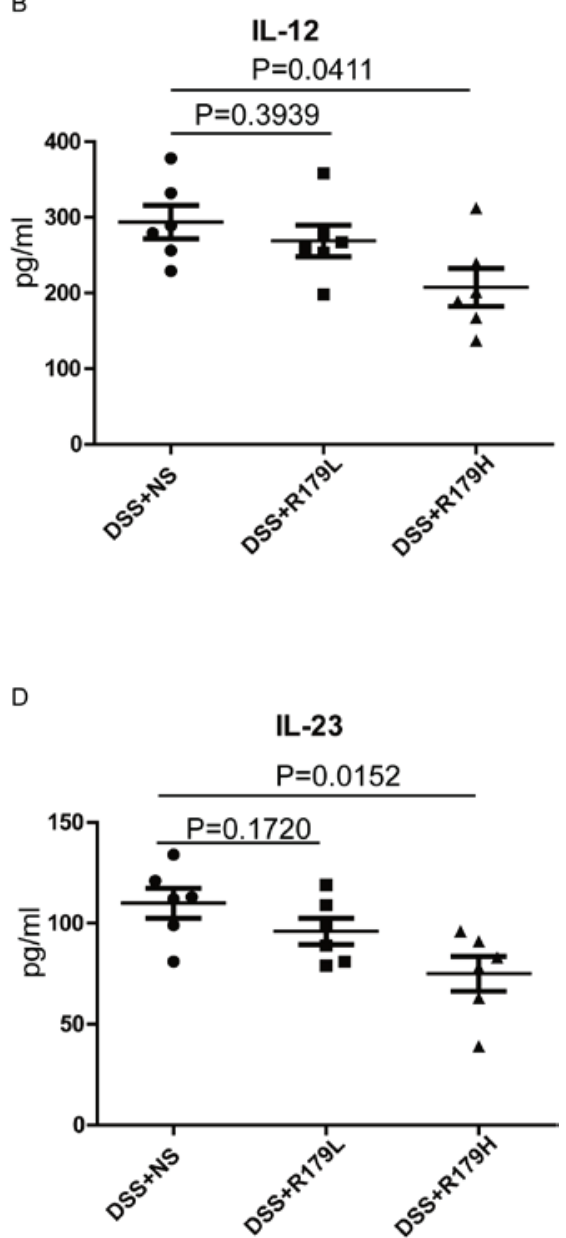

Figure 4. Bacillus subtilis administration alleviates DSS-induced systemic inflammation. Serum (A) IL-10, (B) IL-12, (C) IL-17 and (D) IL-23 detection by ELISA. DSS, dextrose sulfate sodium; NS, normal saline; R179L, low-dose B. subtilis; R179H, high-dose B. subtillis; IL, interleukin.

A

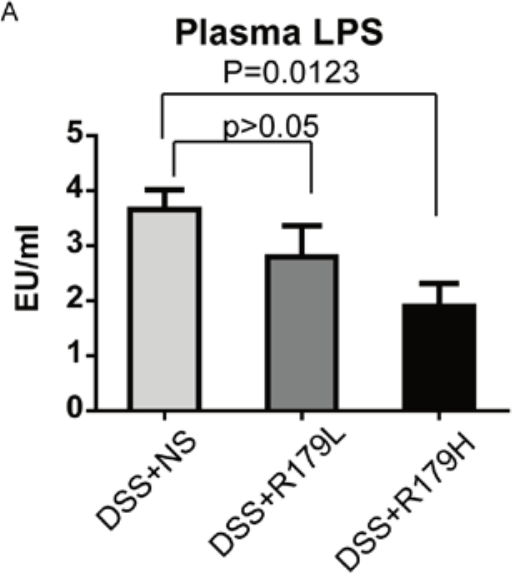

B

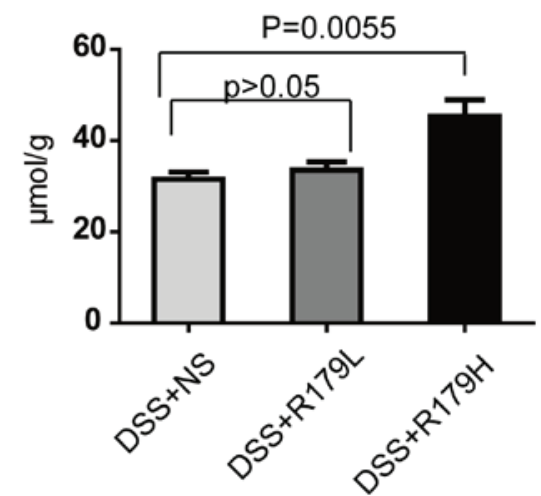

Figure 5. Bacillus subtilis administration balances anti-and pro-inflammatory factors in the gut. Detection of (A) plasma LPS and (B) fecal total SCFA. LPS, lipopolysaccharide endotoxin; SCFA, short chain fatty acid; DSS, dextrose sulfate sodium; NS, normal saline; R179L, low-dose B. subtilis; R179H, high-dose B. subtillis.

compared with the control group ( $\mathrm{P}=0.0055$; Fig. 5B), suggesting that high-dose $B$. subtilis was beneficial in maintaining SCFA content, which in turn reduced gut inflammation.
B. subtilis administration ameliorates DSS-induced dysbiosis in the gut of mice. There are 100 trillion microorganisms housed in the human body. These microorganisms are maintained as commensals on the gut mucosa, and are associated 


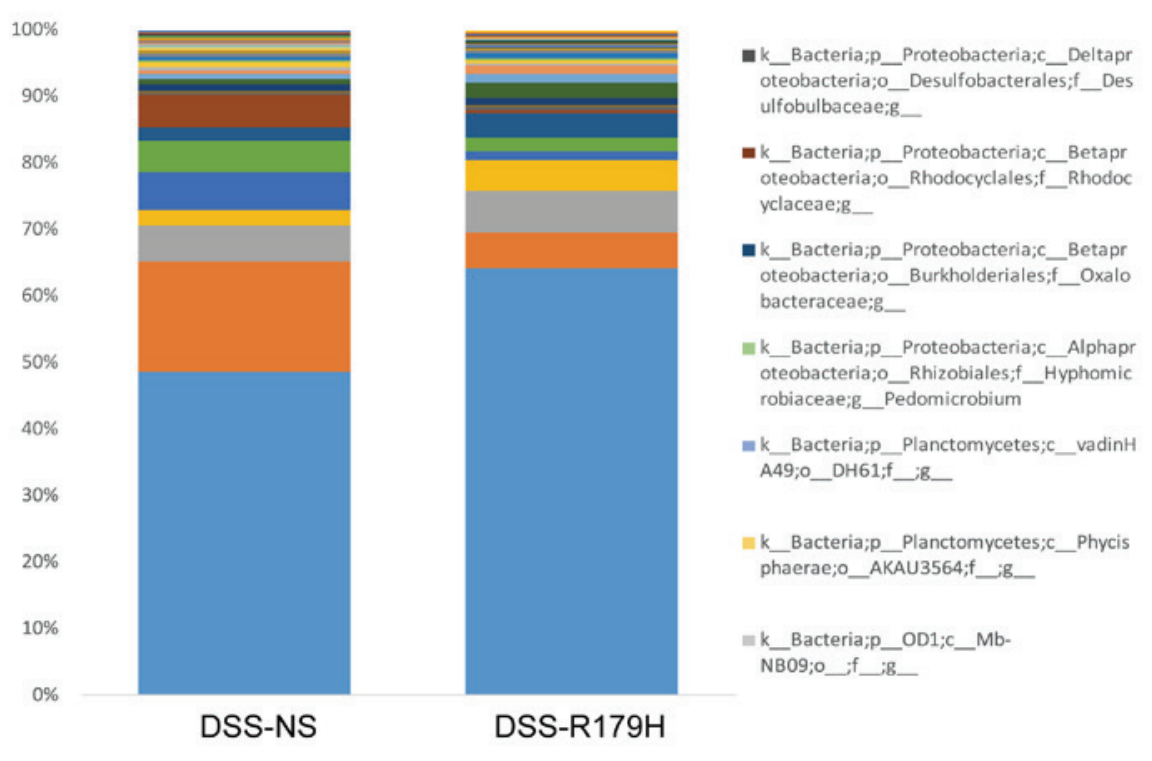

B

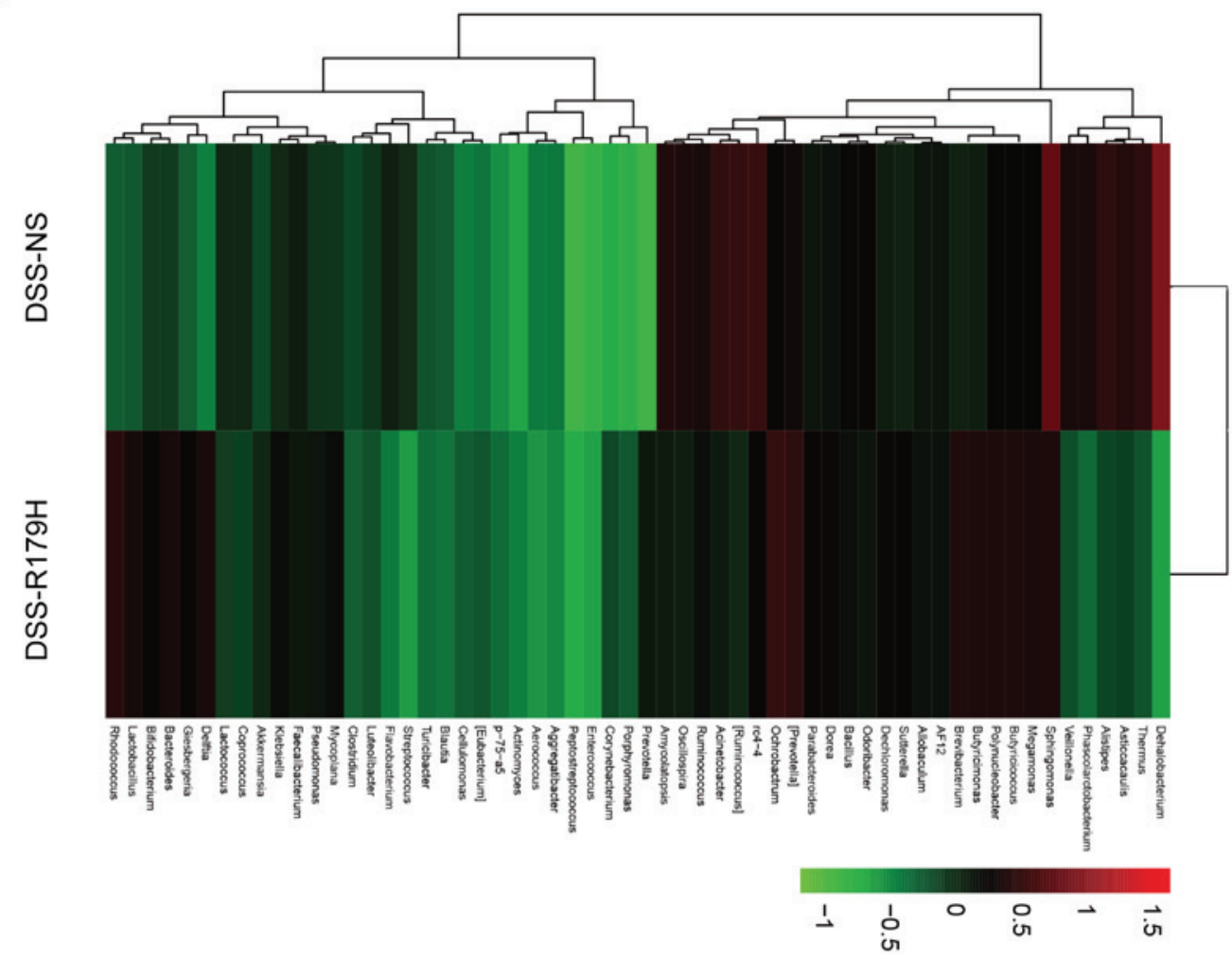

Figure 6. Bacillus subtilis administration ameliorates DSS-induced dysbiosis in the gut. (A) Microbiota composition at the genus level in mice after 4 days of treatment with $B$. subtilis. (B) Heatmap of differentially expressed bacterial genera for NS or high-dose B. subtilis-treated mice (4 days). Mice with the highest and lowest bacterial levels are presented as red and green, respectively. DSS, dextrose sulfate sodium; NS, normal saline; R179H, high-dose B. subtillis.

with metabolism, including maintaining the internal environment and regulating the immune system (27). Consequently, 16s-rDNA sequencing analysis was performed in the present study to examine changes in the microbiota. As demonstrated in Fig. 6, it was observed that the gut microbiota was markedly altered in the high-dose B. subtilis-treated group compared with the control group. Specifically, a reduction of
Acinetobacter sp., Ruminococcus sp., Clostridium spp. and Veillonella sp. was detected upon high-dose B. subtilis treatment, whereas levels of Bifidobacterium sp., Lactobacillus sp., and Butyricicoccus sp. were increased. Acinetobacter spp., Ruminococcus spp. Clostridium sp. and Veillonella sp. have been shown to be overrepresented in IBD patients, whereas Bifidobacterium spp., Lactobacillus spp., and Butyricicoccus sp. 
are decreased (28-32). These results demonstrated the role of B. subtilis in the amelioration of DSS-induced dysbiosis in the gut of model mice.

\section{Discussion}

In the present study, DSS was used to induce UC in a mouse model, and treatment with B. subtilis was revealed to markedly decrease the mortality of DSS-treated mice and protect the intestine from further damage. In addition, $B$. subtilis treatment decreased the damage caused by DSS, which supports the hypothesis that B. subtilis is able to repair epithelial cell injury in intestinal inflammation via immunomodulation (9). Elevated levels of IL-12, IL-17 and IL-23 have previously been found in the epithelial mucosal barrier of subjects with IBD, whereas IL-10 is known to have a protective role in alleviating gut inflammation $(9,33,34)$. Additionally, the present study detected elevated levels of IL-10 and decreased levels of IL-12, IL-17 and IL-23 in the high-dose, but not low-dose, B. subtilis-treatment groups. Therefore, the present study provides evidence that $B$. subtilis regulates gut immune balance in a dose-dependent manner.

A reduction in the number of SCFA-producing bacteria can result in a degree of focal metabolic stress and vulnerability to inflammatory disease (35). Using gas chromatography, it was determined that $B$. subtilis administration increased the levels of SCFAs. In addition, a significant increase of the Butyricicoccus spp., which contributes to butyrate generation (36), was detected upon B. subtilis treatment in the present study. Previous studies have shown that the IBD phenotype was associated with lower levels of the clostridial cluster IV genus Butyricicoccus $(36,37)$. These findings indicate that $B$. subtilis may be beneficial for the survival and expansion of Butyricicoccus spp. under the conditions of gut damage.

A balance of healthy gut commensal bacteria is required for the suppression of pathogenic infections (38), with increasing evidence suggesting that the restoration of normal commensals via transplant is more effective at fighting Clostridium sp. infection than antibiotics (39). Commercially available probiotics, including Lactobacillus and Bifidobacterium spp., are used to attenuate inflammatory activity and prevent relapses in UC (40). In the present study, it was determined that beneficial Bifidobacterium, Lactobacillus, and Butyricicoccus spp. increased in the high-dose B. subtilis-treated groups, compared with the control group. Species known to promote gut damage, such as Acinetobacter sp., Ruminococcus sp., Clostridium spp. and Veillonella sp, were found to be decreased following B. subtilis treatment in the present study. These results indicate the potential role of $B$. subtilis administration in restoring a healthy balance of beneficial and harmful bacteria in the gut.

In conclusion, dose-dependent $B$. subtilis administration was demonstrated to aid intestinal mucosa recovery from DSS-induced damage and protect the intestinal mucosa by balancing beneficial and harmful bacterium and their respective, associated anti- and pro-inflammatory agents. The present study elucidated the mechanisms of $B$. subtilis action and provided preclinical data for $B$. subtilis use in UC therapy.

\section{Acknowledgements}

This study was supported by grants from the Basic Research Program of Beijing Renze Foundation (grant no.ZX-IBD-13002). The authors would like to thank Dong-Ping Hu, Dan-Dan Huang, Shan-Hua Tang, Lin-Na Guo and Dan Cao, Technicians from the International Cooperation Laboratory on Signal Transduction (Eastern Hepatobiliary Surgery Hospital, Second Military Medical University, Shanghai, China), for their technical assistance.

\section{References}

1. Mosli MH, Feagan BG, Sandborn WJ, D'Haens G, Behling C, Kaplan K, Driman DK, Shackelton LM, Baker KA, Macdonald JK, et al: Histologic evaluation of ulcerative colitis: A systematic review of disease activity indices. Inflamm Bowel Dis 20: 564-575, 2014

2. Rosenberg L, Nanda KS, Zenlea T, Gifford A, Lawlor GO, Falchuk KR, Wolf JL, Cheifetz AS, Goldsmith JD and Moss AC: Histologic markers of inflammation in patients with ulcerative colitis in clinical remission. Clin Gastroenterol Hepatol 11: 991-996, 2013.

3. Manichanh C, Borruel N, Casellas F and Guarner F: The gut microbiota in IBD. Nat Rev Gastroenterol Hepatol 9: 599-608, 2012.

4. Conrad K, Roggenbuck D and Laass MW: Diagnosis and classification of ulcerative colitis. Autoimmun Rev 13: 463-466, 2014.

5. Suzuki T: Regulation of intestinal epithelial permeability by tight junctions. Cell Mol Life Sci 70: 631-659, 2013.

6. Buning C, Geissler N, Prager M, Sturm A, Baumgart DC, Büttner J, Bühner S, Haas V and Lochs H: Increased small intestinal permeability in ulcerative colitis: Rather genetic than environmental and a risk factor for extensive disease? Inflamm Bowel Dis 18: 1932-1939, 2012.

7. Shen J, Zuo ZX and Mao AP: Effect of probiotics on inducing remission and maintaining therapy in ulcerative colitis, Crohn's disease, and pouchitis: Meta-analysis of randomized controlled trials. Inflamm Bowel Dis 20: 21-35, 2014.

8. Aly SM, Abdel-Galil Ahmed Y, Abdel-Aziz Ghareeb A and Mohamed MF: Studies on Bacillus subtilis and Lactobacillus acidophilus, as potential probiotics, on the immune response and resistance of Tilapia nilotica (Oreochromis niloticus) to challenge infections. Fish Shellfish Immunol 25: 128-136, 2008.

9. Sarra M, Pallone F, Macdonald TT and Monteleone G: IL-23/IL-17 axis in IBD. Inflamm Bowel Dis 16: 1808-1813, 2010.

10. Nell S, Suerbaum S and Josenhans C: The impact of the microbiota on the pathogenesis of IBD: Lessons from mouse infection models. Nat Rev Microbiol 8: 564-577, 2010.

11. Okamoto K, Fujiya M, Nata T, Ueno N, Inaba Y, Ishikawa C, Ito T, Moriichi K, Tanabe H, Mizukami Y, et al: Competence and sporulation factor derived from Bacillus subtilis improves epithelial cell injury in intestinal inflammation via immunomodulation and cytoprotection. Int J Colorectal Dis 27: 1039-1046, 2012.

12. Selvam R, Maheswari P, Kavitha P, Ravichandran M, Sas B and Ramchand CN: Effect of Bacillus subtilis PB6, a natural probiotic on colon mucosal inflammation and plasma cytokines levels in inflammatory bowel disease. Indian J Biochem Biophys 46: 79-85, 2009.

13. Axelsson LG, Landström E, Goldschmidt TJ, Grönberg A and Bylund-Fellenius AC: Dextran sulfate sodium (DSS) induced experimental colitis in immunodeficient mice: Effects in CD4(+)-cell depleted, athymic and NK-cell depleted SCID mice. Inflamm Res 45: 181-191, 1996.

14. Lindén SK, Florin TH and McGuckin MA: Mucin dynamics in intestinal bacterial infection. PLoS One 3: e3952, 2008.

15. Olson TS, Reuter BK, Scott KG, Morris MA, Wang XM, Hancock LN, Burcin TL, Cohn SM, Ernst PB, Cominelli F, et al: The primary defect in experimental ileitis originates from a nonhematopoietic source. J Exp Med 203: 541-552, 2006.

16. Cole JR, Wang Q, Cardenas E, Fish J, Chai B, Farris RJ, Kulam-Syed-Mohideen AS, McGarrell DM, Marsh T, Garrity GM and Tiedje JM: The ribosomal database project: Improved alignments and new tools for rRNA analysis. Nucleic Acids Res 37 (Database Issue): D141-D145, 2009. 
17. Li W and Godzik A: Cd-hit: A fast program for clustering and comparing large sets of protein or nucleotide sequences. Bioinformatics 22: 1658-1659, 2006.

18. Caporaso JG, Kuczynski J, Stombaugh J, Bittinger K, Bushman FD, Costello EK, Fierer N, Peñna AG, Goodrich JK, Gordon JI, et al: QIIME allows analysis of high-throughput community sequencing data. Nat Methods 7: 335-336, 2010.

19. Chassaing B, Aitken JD, Malleshappa M and Vijay-Kumar M: Dextran sulfate sodium (DSS)-induced colitis in mice. Curr Protoc Immunol 104: Unit 15-25, 2014.

20. Goto Y, Kurashima Y and Kiyono H: Roles of the gut mucosal immune system in symbiosis and immunity. Rinsho Ketsueki 56: 2205-2212, 2015 (In Japanese).

21. Genser L, Poitou C, Brot-Laroche É, Rousset M, Vaillant JC, Clément $\mathrm{K}$, Thenet $\mathrm{S}$ and Leturque A: Alteration of intestinal permeability: The missing link between gut microbiota modifications and inflammation in obesity? Med Sci (Paris) 32: 461-469, 2016 (In French).

22. Fujino S, Andoh A, Bamba S, Ogawa A, Hata K, Araki Y, Bamba T and Fujiyama Y: Increased expression of interleukin 17 in inflammatory bowel disease. Gut 52: 65-70, 2003.

23. Calvano SE, Xiao W, Richards DR, Felciano RM, Baker HV, Cho RJ, Chen RO, Brownstein BH, Cobb JP, Tschoeke SK, et al: A network-based analysis of systemic inflammation in humans. Nature 437: 1032-1037, 2005.

24. Cetin S, Ford HR, Sysko LR, Agarwal C, Wang J, Neal MD, Baty C, Apodaca G and Hackam DJ: Endotoxin inhibits intestinal epithelial restitution through activation of Rho-GTPase and increased focal adhesions. J Biol Chem 279: 24592-24600, 2004.

25. Maslowski KM, Vieira AT, Ng A, Kranich J, Sierro F, Yu D, Schilter HC, Rolph MS, Mackay F, Artis D, et al: Regulation of inflammatory responses by gut microbiota and chemoattractant receptor GPR43. Nature 461: 1282-1286, 2009.

26. Mirmonsef P, Zariffard MR, Gilbert D, Makinde H, Landay AL and Spear GT: Short-chain fatty acids induce pro-inflammatory cytokine production alone and in combination with toll-like receptor ligands. Am J Reprod Immunol 67: 391-400, 2012.

27. Magri G and Cerutti A: A touch of youth in gut microbiota development. Immunity 45: 12-14, 2016.

28. Bergogne-Bérézin E and Towner KJ: Acinetobacter spp. as nosocomial pathogens: Microbiological, clinical, and epidemiological features. Clin Microbiol Rev 9: 148-165, 1996.

29. Sokol H, Leducq V, Aschard H, Pham HP, Jegou S, Landman C, Cohen D, Liguori G, Bourrier A, Nion-Larmurier I, et al: Fungal microbiota dysbiosis in IBD. Gut: pii: gutjnl-2015-310746, 2016.
30. Nones K, Knoch B, Dommels YE, Paturi G, Butts C, McNabb WC and Roy NC: Multidrug resistance gene deficient (mdr1a-/-) mice have an altered caecal microbiota that precedes the onset of intestinal inflammation. J Appl Microbiol 107: 557-566, 2009.

31. Kummen M, Holm K, Anmarkrud JA, Nygård S, Vesterhus M, Høivik ML, Trøseid M, Marschall HU, Schrumpf E, Moum B, et al: The gut microbial profile in patients with primary sclerosing cholangitis is distinct from patients with ulcerative colitis without biliary disease and healthy controls. Gut: pii: gutjnl-2015-310500, 2016.

32. Gevers D, Kugathasan S, Denson LA, Vázquez-Baeza Y, Van Treuren W, Ren B, Schwager E, Knights D, Song SJ, Yassour M, et al: The treatment-naive microbiome in new-onset Crohn's disease. Cell Host Microbe 15: 382-392, 2014.

33. Schreiber S, Heinig T, Thiele HG and Raedler A: Immunoregulatory role of interleukin 10 in patients with inflammatory bowel disease. Gastroenterology 108: 1434-1444, 1995.

34. Steinbach EC and Plevy SE: The role of macrophages and dendritic cells in the initiation of inflammation in IBD. Inflamm Bowel Dis 20: 166-175, 2014.

35. Schoultz I, Söderholm JD and McKay DM: Is metabolic stress a common denominator in inflammatory bowel disease? Inflamm Bowel Dis 17: 2008-2018, 2011.

36. Eeckhaut V, Machiels K, Perrier C, Romero C, Maes S, Flahou B, Steppe M, Haesebrouck F, Sas B, Ducatelle R, et al: Butyricicoccus pullicaecorum in inflammatory bowel disease. Gut 62 : 1745-1752, 2013

37. Frank DN, Robertson CE, Hamm CM, Kpadeh Z, Zhang T, Chen H, Zhu W, Sartor RB, Boedeker EC, Harpaz N, et al: Disease phenotype and genotype are associated with shifts in intestinal-associated microbiota in inflammatory bowel diseases. Inflamm Bowel Dis 17: 179-184, 2011.

38. Kamada N, Kim YG, Sham HP, Vallance BA, Puente JL, Martens EC and Núñez G: Regulated virulence controls the ability of a pathogen to compete with the gut microbiota. Science 336: 1325-1329, 2012.

39. van Nood E, Vrieze A, Nieuwdorp M, Fuentes S, Zoetendal EG, de Vos WM, Visser CE, Kuijper EJ, Bartelsman JF, Tijssen JG, et al: Duodenal infusion of donor feces for recurrent Clostridium difficile. N Engl J Med 368: 407-415, 2013.

40. Meijer BJ and Dieleman LA: Probiotics in the treatment of human inflammatory bowel diseases: Update 2011. J Clin Gastroenterol 45 (Suppl): S139-S144, 2011. 